\title{
El empleo de las personas con discapacidad en la gran recesión: ¿Son los Centros Especiales de Empleo una excepción?
}

\author{
VANESA RODRÍGUEZ ÁLVAREZ \\ Departamento de Sociología, UNIVERSIDAD DE OVIEDO, ESPAÑA. E-mail: \\ rodriguezvanesa@uniovi.es
}

\begin{abstract}
RESUMEN
En un contexto como el actual de crisis económica con fuertes caídas en la contratación, los contratos celebrados con personas con discapacidad han experimentado un fuerte incremento debido al aumento de la contratación temporal en los Centros Especiales de Empleo, mientras que los contratos a personas con discapacidad en empresas ordinarias han sufrido un retroceso superior al de los contratos de los no discapacitados. Estos datos muestran una tendencia hacia el crecimiento del empleo protegido en detrimento del empleo ordinario, situación contraria a la expuesta como idónea en la Ley de Integración Social de los Minusválidos, aunque parece indicar que los CEE actúan como "empleo refugio" de las personas con discapacidad en momentos de crisis.
\end{abstract}

Palabras clave: Discapacidad, empleo protegido, empleo ordinario.

\section{The Employment of People with Disabilities in the Great Recession: Are the Sheltered Employment Centers an Exception?}

\begin{abstract}
The current economic crisis has reduced substantially the number of employment. However, statistics from the Public Employment Service shows an increase in the number of jobs contracts signed by disabled people. The main reason in that Sheltered Employment Centers have raised largely their contracting. On the opposite, ordinary firms have reduced hiring with disabled workers. These data seem to show an upward trend in Sheltered Employment Centers while disabled people in regular employment diminishes. This conclusion is against the aim of the Social Integration for Disabled People Act, although it seems that the SEC's acting as "employment refuge" of disabled people in times of crisis.
\end{abstract}

Keywords: Disability, Sheltered Employment, Ordinary Employment.

Clasificación JEL: J14, J41

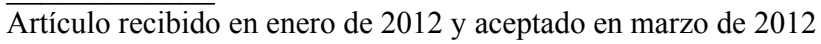

Artículo disponible en versión electrónica en la página www.revista-eea.net, ref. ə-30106 


\section{INTRODUCCIÓN}

En periodos de bonanza económica no todos los colectivos aumentan su participación en el mercado de trabajo por igual. Asimismo, las crisis en el mercado de trabajo no golpean a todos sus integrantes de la misma manera. En España desde el último trimestre de 2007 hasta el último de 2011 la crisis económica implicó una pérdida de empleo de aproximadamente 2.500 .000 ocupados y que la tasa de paro haya aumentado en más de un 14 por ciento desde el inicio de la crisis situándose a finales de 2011 en un 22,85 por ciento.

La reducción del empleo fue menos intensa entre los asalariados que entre los trabajadores por cuenta propia. Si analizamos el empleo desde una perspectiva de género, observamos una evolución menos negativa del empleo femenino que la del masculino. Sin embargo, ésto se debe más a un incremento del paro masculino (el empleo masculino está muy relacionado con la construcción y la industria que son los sectores más afectados por el aumento del paro) que a una buena situación del empleo femenino. La reducción del empleo ha afectado más profundamente a la población con bajo nivel formativo. En cuanto a la nacionalidad, la tasa de paro de los inmigrantes supera en más de 14 puntos a la de nacionalidad española.

Diversos trabajos han analizado los efectos de la crisis según la nacionalidad, el género o el nivel formativo (Medina et al., 2010; IVIE, 2010). Sin embargo a pesar de que a priori esta situación de crisis económica probablemente ha debido golpear fuertemente al empleo de las personas con discapacidad, debido a su bajo nivel formativo, su alta temporalidad y su mayor tasa de ocupación masculina, no se han llevado a cabo análisis que profundicen en la situación actual de dicho colectivo.

Esta ausencia de estudios puede estar explicada por la escasez de fuentes de información sobre discapacidad y empleo. El objetivo de este artículo, y esto constituye una novedad respecto a la literatura existente, es realizar una aproximación a los efectos de la crisis en la contratación de las personas con discapacidad, teniendo en cuenta si esta contratación se ha llevado a cabo en una empresa ordinaria o en un Centro Especial de Empleo (CEE). Para ello nos basaremos en el registro de contratos del Servicio Público de Empleo que proporciona información sobre los contratos celebrados con individuos que comunican una discapacidad, independientemente del hecho de que sea un contrato específico para personas con discapacidad o no. Por lo tanto, este análisis, se basa en fuentes administrativas y, por tanto, hace referencia únicamente a las personas con discapacidad que están en posesión de un Certificado de minusvalía. En este sentido, cabe señalar que Malo (2003) considera que los datos de fuentes administrativas (en función de la posesión o no del Certificado de minusvalía) relacionan el concepto de discapacidad como discapacidad para trabajar lo que dificulta el análisis de la influencia de la discapacidad en la partici- 
pación laboral. Esta limitación de la base de datos utilizada, sobre otras más genéricas sobre discapacidad ${ }^{1}$ (EDDES-1999 y EDAD-2008), es compensada por el hecho de que en este trabajo no se pretende analizar la participación laboral de las personas con discapacidad, sino su contratación. Al utilizar como referencia el Certificado de minusvalía estamos centrándonos en aquellas personas con discapacidad que potencialmente pueden ser objeto de medidas de fomento del empleo para este colectivo, ya sea mediante contratación en un CEE o mediante contratos específicos para este colectivo; por lo tanto, podemos medir en mayor medida cual es el peso que este tipo de empleo tiene entre las personas con discapacidad.

\section{LAS PERSONAS CON DISCAPACIDAD Y SU RELACIÓN CON EL MERCADO DE TRABAJO}

A pesar de que en la actualidad se ve la integración laboral de las personas con discapacidad como una vía para conseguir el desarrollo pleno de su personalidad, a través de la independencia personal y económica que otorga la participación activa en el mercado de trabajo, esto no siempre ha sido así. En los últimos años se ha pasado de una política sobre discapacidad centrada en la garantía de ingresos hacia una política que ve el empleo como la principal herramienta de integración social de este colectivo.

Este cambio en la percepción de la importancia del empleo para las personas con discapacidad se aprecia también en la literatura académica; los estudios sobre discapacidad y mercado de trabajo son relativamente recientes, especialmente en el caso español. En los años 70 comienzan a aparecer estudios empíricos sobre la participación laboral de los discapacitados en Estados Unidos, a partir de la década de los 90 se empiezan a llevar a cabo estudios de este tipo en el ámbito europeo y a comienzos de este siglo aparecen los primeros estudios realizados en España (Baldwin y Johnson, 1992; Baldwin y Schumacher, 2002: Gannon y Nolan, 2004).

Todos estos estudios coinciden en que tener alguna discapacidad afecta negativamente a la participación laboral, tanto en la tasa de paro como en la de actividad; y que estas diferencias en participación con las personas sin discapacidad no se pueden explicar completamente por diferencias en características, es decir, hay una parte debida a discriminación hacia las personas con discapacidad y/o a diferencias de productividad no observadas correlacionadas con la discapacidad.

Existe consenso en la literatura sobre que la participación laboral no es homogénea dentro del grupo de personas con discapacidad. Variables como el tipo

\footnotetext{
${ }^{1}$ Un análisis más completo de las características básicas que debe tener una definición de discapacidad desde el punto de vista económico se puede consultar en Malo (2007).
} 
de discapacidad, el género o la inversión en capital humano influyen en la probabilidad de participar en el mercado de trabajo de forma negativa y positiva respectivamente (Zwinkels, 2001, Dávila, 2003; Villa, 2003: Dávila y Malo, 2006; Dávila, 2006 y Malo et al., 2009). Respecto al nivel educativo de las personas con discapacidad, Dávila (2003) constata la existencia de una brecha entre los niveles educativos de la población joven discapacitada y la población joven en general: el analfabetismo es veinte veces mayor entre los jóvenes discapacitados que entre los jóvenes en general, los porcentajes de jóvenes con Estudios Primarios son similares en ambos colectivos y, respecto a los Estudios Secundarios o Universitarios, los porcentajes de jóvenes no discapacitados duplican y cuadriplican a los de discapacitados. Jones et al. (2007) encuentran diferencias importantes de participación en el mercado de trabajo entre aquellos individuos que han nacido con una discapacidad y aquellos que pasan a ser discapacitados en un momento posterior. Este último colectivo es más sensible a los efectos negativos de la discapacidad sobre la participación en el mercado de trabajo; principalmente aquellos individuos con un bajo nivel de capital humano.

La investigación sobre el caso español muestra con claridad que el principal problema de las personas con discapacidad es su baja participación laboral. Así, Malo (2001, 2003, 2004), Dávila (2006), Malo et al. (2009) y Cueto et al. (2012) muestran que la tasa de actividad de las personas con discapacidad alcanza valores cercanos a la mitad de la tasa de actividad de los no discapacitados.

Además, Cueto et al. (2012) al comparar los resultados obtenidos con la EDAD-2008 con los de la EDDES-1999 concluyen que las tasas de actividad y de empleo de las personas con discapacidad son la mitad que las de las no discapacitadas; mientras que la tasa de paro es el doble, situación que apenas ha variado respecto a la registrada hace una década. Entre ambas encuestas la tasa de actividad de la población discapacitada se ha incrementado en apenas dos puntos frente al aumento entre la población sin discapacidad que ha sido de 7 puntos; la tasa de ocupación de las personas con discapacidad aumentó 3 puntos, mientras que el incremento en la población sin discapacidad ha llegado a casi diez puntos porcentuales.

Una vez que las personas con discapacidad se incorporan al mercado de trabajo su ocupación se centra en trabajos a tiempo parcial y menos remunerados que los de los no discapacitados, además de ser más probable que sufran desempleo o inactividad. (Zwinkels, 2001; Pascual y Cantarero, 2007; Pagán, 2012).

Respecto al análisis de la satisfacción laboral, ésta es superior en el caso de las personas con discapacidad, posiblemente debido a que las expectativas sobre el empleo de las personas con discapacidad son más bajas que las de sin discapacidad. (Pagán y Malo, 2009; Pagán, 2011a). Sin embargo, según Pagán 
(2011b) en el caso de autoempleo no se encuentra evidencia de que la relación entre autoempleo y satisfacción en el trabajo difiera entre personas mayores con discapacidad y sin discapacidad.

Los menores salarios de las personas con discapacidad son un tema recurrente en los trabajos que analizan la relación entre discapacidad y mercado de trabajo, aunque muchos de ellos no entran a evaluar a que se deben estas diferencias salariales (Jones et al., 2006; Humer et al., 2007 y Brown y Emery, 2008). Por otro lado, la mayoría de los trabajos que analizan esta diferencia salarial observan que una parte de ella no se puede explicar por diferencias en características (Johnson y Lambrinos, 1985; Baldwin y Johnson, 2000 y Malo y Pagán, 2012). Aunque tradicionalmente se relaciona el componente rendimientos con la discriminación, en el caso de las personas con discapacidad se debe interpretar con precaución debido a que sus características pueden limitar realmente su productividad (Baldwin y Johnson, 1995; Malo y Pagán, 2012).

Otros estudios más recientes analizan la relación entre empleo y discapacidad desde un punto de vista dinámico. Gannon y Nolan (2007) realizan un análisis de las transiciones dentro y fuera de la discapacidad y la relación de estas con el empleo. Estos autores cuantifican el impacto del inicio, la perseverancia y la salida de la discapacidad, encontrando que la probabilidad de ser activo se reduce en un 20 y en un $42 \%$ y se incrementa en un $7 \%$ respectivamente. Pagán (2007) no encuentra diferencias importantes en las trayectorias de discapacidad entre hombres y mujeres. En cuanto a la duración de la discapacidad concluye que los primeros años disminuye el porcentaje de personas discapacitadas, pero que después de estos años la tasa de disminución de la discapacidad se reduce; y que son los individuos de mayor edad y con un nivel educativo más bajo los que tienen las mayores probabilidades de permanecer en la discapacidad.

Malo y Muñoz-Bullón (2006) llevan a cabo una evaluación de los efectos de las políticas de promoción del empleo de las personas con discapacidad sobre la calidad del emparejamiento con el puesto de trabajo, encontrando que los servicios de intermediación no aumentan la calidad del empleo.

\subsection{El empleo en los Centros Especiales de Empleo}

En el actual marco en el que se encuadran las medidas dirigidas a la integración sociolaboral de las personas con discapacidad en España, los Centros especiales de empleo (CEE) son, desde hace ya más de dos décadas, uno de los pilares clave de éstas. Esta actuación no ha estado exenta de polémica, con opiniones tanto a favor como en contra. En cierta medida, la Estrategia Global de Acción para el Empleo de Personas con Discapacidad reconoce ambos puntos de vista puesto que plantea la renovación de los CEE, tratando de mantener 
la generación de empleo pero, a la vez, favoreciendo el tránsito al empleo ordinario.

Los argumentos a favor se centran en que los CEE contribuyen a reducir la inactividad de este colectivo además de proporcionales un trabajo remunerado, favoreciendo su desarrollo personal. Estos centros proporcionan experiencia laboral, formación y capacitación profesional; sus trabajadores adquieren habilidades y hábitos laborales y adquieren cierta polivalencia y flexibilidad (características valoradas en el mercado de trabajo ordinario); proporcionan a las personas con discapacidad formación, empleo y hábitos laborales (Rubio, 2003).

Los argumentos en contra señalan que los CEE, en lugar de integrar, pueden segregar, en la medida en que constituyen un punto de destino y no un medio de tránsito al empleo en el mercado de trabajo ordinario (las tasas de transición son muy bajas, entre el 1 y el 5 por ciento); que su objetivo es la productividad y competitividad pero en condiciones laborales precarias y sin sindicación; que no se aplican los convenios colectivos del sector o rama al que pertenecen ${ }^{2}$; la inexistencia de representación sindical; que el trabajo se desarrolla en condiciones de sobreprotección y menor exigencia a las de las empresas ordinarias, de manera que los trabajadores con discapacidad no adquieren las capacidades necesarias para desenvolverse en el mercado de trabajo normal; que en la mayor parte de países los salarios son bajos, hay una pobre protección laboral y el estado del trabajador es incierto (Rubio, 2003; OCDE, 1992).

Así, López y Seco (2005) hacen referencia a una posible precarización laboral e inestabilidad del colectivo como consecuencia del comportamiento oportunista de algunos empresarios que se aprovechan de la explosión del número de CEE, y al importante número de bajas de entidades, que no llaman tanto la atención debido a que el número de nuevas altas es superior.

En cuanto a la composición de los CEE, según CCOO $(2009,2010)$ el 99\% de los trabajadores con discapacidad en CEE están contratados bajo la figura de operario; por lo que según el convenio colectivo de aplicación le corresponde un salario igual al SMI. También indican que en los CEE el 70 por ciento de los trabajadores cobra el $\mathrm{SMI}^{3}$, el 15 por ciento de los trabajadores cobran entre 700 y 800 euros y el 10 por ciento no alcanza los 900 euros. Por otro lado Villa (2003) con datos de la EDDES-1999 encuentra que el empleo protegido en CEE era la medida de fomento del empleo de personas con discapacidad más utili-

\footnotetext{
${ }^{2}$ Según Esteban et al. (2009) según el Principio de Especialidad, en el caso que concurran convenios colectivos supraempresariales específicos de CEE con convenios colectivos sectoriales (lo que ocurre desde 2003 con la aprobación del XI Convenio colectivo de centros de asistencia, atención, diagnóstico y promoción de personas con discapacidad, que incluye en su ámbito de aplicación) deben aplicarse los primeros.

3 Esta diferencia se puede explicar por los convenios colectivos de empresa o los acuerdos individuales que mejoran las condiciones de trabajo.
} 
zada por los hombres, y la segunda más utilizada por las mujeres, suponiendo en total la medida que beneficiaba a mayor número de trabajadores. También contrasta estos resultados a partir de la Estadística de contratos del INEM para el periodo comprendido entre 1996 y 2001, sin embargo no profundiza más en el análisis de estos centros.

En general, aunque la evidencia empírica es escasa, hay consenso entre los autores sobre el escaso impacto que el empleo protegido tiene como forma de inserción en el mercado laboral ordinario (Cueto, et al., 2008; López y Seco, 2005; Delsen, 2001; Visier, 1998).

\section{EVOLUCIÓN DE LA CONTRATACIÓN DE LAS PERSONAS CON DISCAPACIDAD}

Según datos del INE (Gráfico 3.1), la tasa de actividad de las personas con discapacidad en 2010 era de aproximadamente la mitad que la tasa de actividad de los no discapacitados, aunque al igual que ésta ha aumentado desde 2008 a pesar del escenario de crisis económica ${ }^{4}$. Al igual que para el total de la población, la tasa de actividad de los hombres con discapacidad supera a la de las mujeres, sin embargo la brecha entre las tasas de actividad de ambos sexos siempre ha sido más reducida en el caso de las personas con discapacidad.

Gráfico 3.1.

Evolución de las tasas de actividad y empleo según discapacidad (2008-2010)

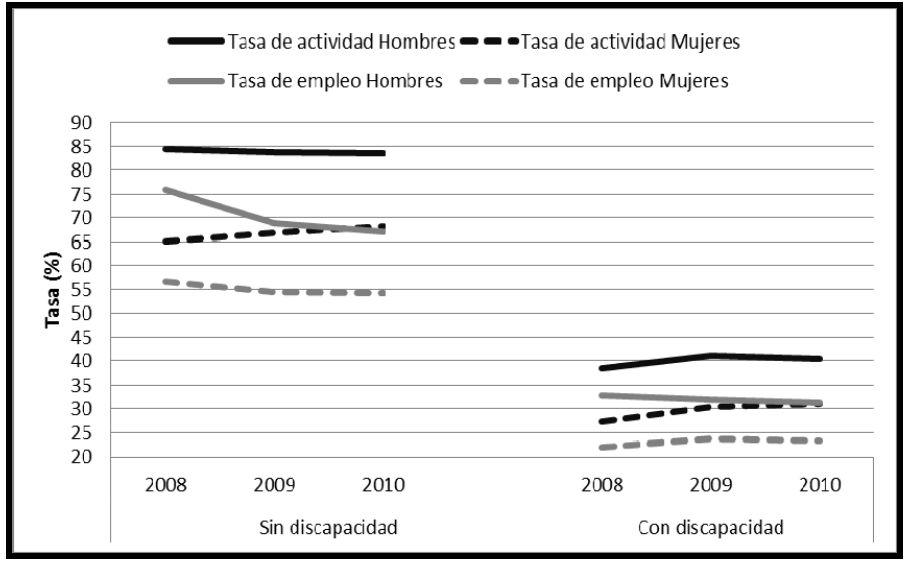

Fuente: INE.

\footnotetext{
${ }^{4}$ Los datos proporcionados por el INE sobre el empleo de las personas con discapacidad únicamente están referidos al periodo 2008-2010. Estos datos son el resultado de cruzar la información de la Encuesta de Población Activa (EPA) del INE con la de la Base de Datos Estatal de Personas con Discapacidad (BEPD) del IMSERSO.
} 
La tasa de empleo de las personas con discapacidad es menos de la mitad de la de los no discapacitados. Aunque en los últimos años esta tasa ha disminuido en ambos colectivos, la diferencia entre ambas ha aumentado. Al igual que para el total de la población, las diferencias entre las tasas de empleo de los hombres y mujeres con discapacidad se han reducido desde el comienzo de la crisis.

En cuanto a la tasa de paro (Gráfico 3.2), la brecha existente entre las personas con y sin discapacidad se ha reducido considerablemente en los últimos años, de casi el doble a poco más de 2 puntos porcentuales. Además, al igual que ocurre con las personas no discapacitadas la tasa de paro de las mujeres con discapacidad supera a la de los hombres con discapacidad, aunque en 2010 las diferencias son mayores que en el caso de los no discapacitados.

\section{Gráfico 3.2.}

Evolución de la tasa de paro según discapacidad (2008-2010)

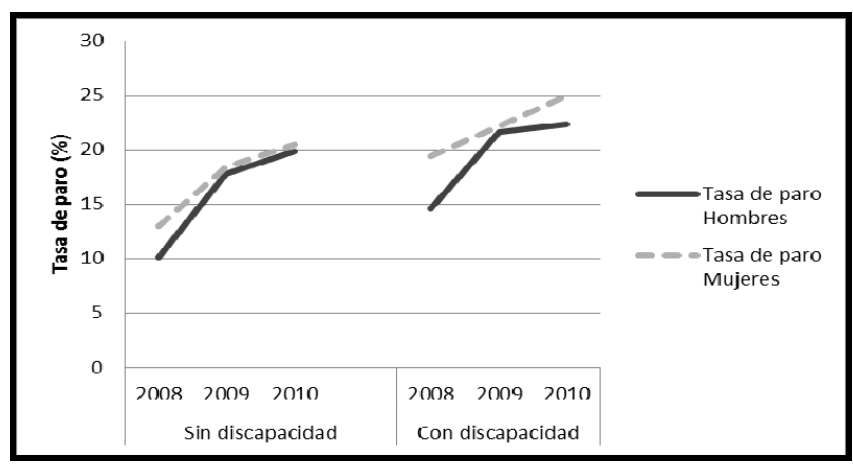

Fuente: INE.

Esto nos podría llevar a pensar que aunque el problema de baja participación de las personas con discapacidad se mantiene, al menos ha mejorado su situación de desempleo respecto a los no discapacitados. No obstante, esta mejora no es debida a una mejora del empleo de las personas con discapacidad, sino a un empeoramiento de las cifras del paro de los no discapacitados. Sin embargo, se debe señalar que el incremento del paro en el colectivo de personas con discapacidad se ha ralentizado más que en otros colectivos. Para analizar con más detalle qué ha pasado con la contratación a las personas con discapacidad se utilizarán datos sobre los contratos registrados.

Según los datos de contratos registrados por el Servicio Público de Empleo Estatal (SPEE), durante los últimos seis años se han realizado más de 50.000 
contratos anuales a personas con discapacidad (siendo entre el 60 y el 76 por ciento de ellos temporales) ${ }^{5}$.

En el Gráfico 3.3 (en el que se muestra la evolución de la contratación como un índice con base 100; es decir usando los datos del año 2006 como referencia) podemos observar que mientras que desde 2007 los contratos celebrados con personas sin discapacidad han disminuido, tanto los de duración determinada como los indefinidos (estos últimos en mayor medida), no ha ocurrido lo mismo con los contratos celebrados con personas con discapacidad, que tras una pequeña bajada entre 2007 y 2009 han crecido casi 20 puntos entre 2009 y 2010 y en 2011 se han mantenido bastante constantes. Este comportamiento positivo de la contratación de personas con discapacidad se debe al incremento de la contratación temporal de este colectivo, que ha aumentado en más de 35 puntos entre 2006 y 2010. Cabe señalar que mientras los contratos temporales de trabajadores no discapacitados se reducían (2007-2009) la contratación temporal de trabajadores con discapacidad se mantenía constante y cuando los contratos temporales de los no discapacitados se estabilizaron, empezaron a incrementarse de forma significativa los contratos temporales a personas con discapacidad. Los contratos indefinidos de personas con discapacidad han seguido una evolución similar a la de los contratos temporales de los no discapacitados, aun con valores negativos no alcanzan la disminución de contratos indefinidos de los no discapacitados.

Gráfico 3.3.

Evolución de los contratos según duración y relación con la discapacidad (Base 100, 2006)

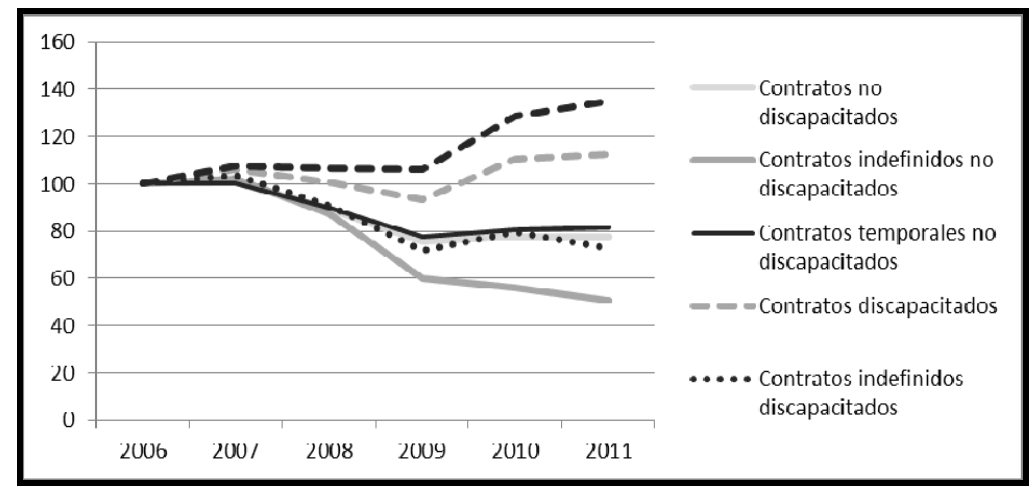

Fuente: SPEE.

\footnotetext{
${ }^{5}$ A partir de este momento los datos proporcionados por el Servicio Público de Empleo no permiten desagregar el análisis por sexos, únicamente proporcionan así desagregados los contratos específicos para personas con discapacidad, y no para el total de contratos celebrados con personas con discapacidad o en CEE.
} 
Se podría pensar que este aumento de la contratación temporal y menor descalabro de la indefinida se debe a la utilización de medidas de fomento para incentivar la contratación de este colectivo (contratos indefinidos y temporales para la contratación de personas con discapacidad). Sin embargo, el uso de los contratos específicos para personas con discapacidad respecto a otros contratos ha descendido en los últimos años. En el Cuadro 3.1 podemos observar como la proporción de contratos tanto indefinidos como temporales específicos para minusválidos ha disminuido casi 10 puntos entre 2006 y 2011. Por tanto, aunque la contratación de personas con discapacidad ha seguido una tendencia ascendente en los últimos años, el uso de contratos específicos para este colectivo ha perdido fuerza en la contratación. Parece que las bonificaciones a la contratación de personas con discapacidad no están logrando el efecto deseado de incentivar la contratación en momentos de crisis en las que la ayuda económica que supone este tipo de bonificación podría ser de gran utilidad. Se debe tener en cuenta que este tipo de contratos bonificados está ligado a unas condiciones de mantenimiento del empleo que en época de crisis pueden no resultar atractivos; en el caso de contratos indefinidos las empresas beneficiarias deben mantener la estabilidad en el empleo de estos trabajadores por un tiempo mínimo de tres años, no pudiendo despedirlos sin causa justificada y, en caso de despido procedente, deberán sustituirlos por otros trabajadores con discapacidad, beneficiándose en este caso solamente de la bonificación de la cuota a la Seguridad Social por los sustitutos; y en el caso de los temporales, estos tienen una duración de entre 12 meses y 3 años.

\section{Cuadro 3.1.}

Porcentaje que los contratos específicos para PCD suponen sobre el total de contratos a PCD

\begin{tabular}{|c|c|c|}
\hline & $\begin{array}{c}\text { \% contratos } \\
\text { específicos/indefinidos }\end{array}$ & $\begin{array}{c}\text { \% contratos } \\
\text { específicos/temporales }\end{array}$ \\
\hline 2006 & 57,9 & 42,3 \\
\hline 2007 & 62,1 & 44,6 \\
\hline 2008 & 56,3 & 41,2 \\
\hline 2009 & 54,6 & 35,5 \\
\hline 2010 & 50,5 & 32,2 \\
\hline 2011 & 48,3 & 32,6 \\
\hline
\end{tabular}

Fuente: SPEE.

Si concretamos un poco más en el análisis del tipo de contrato utilizado, Cuadro 3.2, observamos que hasta 2010 los mayores incrementos en la contratación eran para el contrato eventual por circunstancias de la producción, que 
sufrió un fuerte retroceso en 2011, seguido del contrato de interinidad que experimenta un importante ascenso a partir de 2009. En 2011, los contratos indefinidos, independientemente de su modalidad, presentan un considerable descenso siendo este el primer año desde el inicio de la crisis en el que la contratación indefinida disminuye en todas sus modalidades (excepto la conversión de temporal en indefinido).

Cuadro 3.2.

Evolución de los contratos con personas con discapacidad según tipo

\begin{tabular}{|c|c|c|c|c|c|c|c|c|c|c|}
\hline \multirow{2}{*}{ Tipo de contrato } & \multicolumn{2}{|c|}{2007} & \multicolumn{2}{|c|}{2008} & \multicolumn{2}{|c|}{2009} & \multicolumn{2}{|c|}{2010} & \multicolumn{2}{|c|}{2011} \\
\hline & Total & $\begin{array}{c}\text { Variación } \\
\text { interanual } \\
(\%)\end{array}$ & Total & $\begin{array}{c}\text { Variación } \\
\text { interanual } \\
(\%)\end{array}$ & Total & $\begin{array}{c}\text { Variación } \\
\text { interanual } \\
(\%)\end{array}$ & Total & $\begin{array}{c}\text { Variación } \\
\text { interanual } \\
(\%)\end{array}$ & Total & $\begin{array}{c}\text { Variación } \\
\text { interanual } \\
(\%)\end{array}$ \\
\hline $\begin{array}{l}\text { Indefinido ordinario } \\
\text { (bonif. / no bonif.) }\end{array}$ & 832 & - & 728 & $-12,5$ & 751 & 3,2 & 698 & $-7,1$ & 606 & $-13,2$ \\
\hline $\begin{array}{l}\text { Indefinido fomento } \\
\text { empleo }\end{array}$ & 2.831 & $-6,8$ & 3.082 & 8,9 & 2.323 & $-24,6$ & 2.391 & 2,9 & 1.923 & $-19,6$ \\
\hline Minusválidos & 13.096 & 11,0 & 10.431 & $-20,3$ & 7.966 & $-23,6$ & 8.185 & 2,7 & 7.166 & $-12,4$ \\
\hline Obra o servicio & 7.789 & 3,4 & 7.665 & $-1,6$ & 7.316 & $-4,6$ & 10.416 & 42,4 & 11.334 & 8,8 \\
\hline $\begin{array}{l}\text { Eventual } \\
\text { circunstancias } \\
\text { producción }\end{array}$ & 6.593 & 3,8 & 7.436 & 12,8 & 8.445 & 13,6 & 10.483 & 24,1 & 8.770 & $-16,3$ \\
\hline Interinidad & 5.696 & 14,4 & 6.074 & 6,6 & 7.334 & 20,7 & 8.993 & 22,6 & 11.374 & 26,5 \\
\hline $\begin{array}{l}\text { Temporal } \\
\text { minusválidos }\end{array}$ & 16.739 & 13,3 & 15.327 & $-8,4$ & 13.135 & $-14,3$ & 14.485 & 10,3 & 15.416 & 6,4 \\
\hline Prácticas & 20 & 17,6 & 7 & $-65,0$ & 6 & $-14,3$ & 20 & 233,3 & 17 & $-15,0$ \\
\hline Formación & 608 & $-49,6$ & 563 & $-7,4$ & 620 & 10,1 & 421 & $-32,1$ & 262 & $-37,8$ \\
\hline Resto contratos & 81 & $-8,0$ & 98 & 21,0 & 132 & 34,7 & 111 & $-15,9$ & 84 & $-24,3$ \\
\hline $\begin{array}{l}\text { Convertidos en } \\
\text { indefinidos }\end{array}$ & 4.331 & $-21,8$ & 4.303 & $-0,6$ & 3.549 & $-17,5$ & 4.925 & 38,8 & 5.132 & 4,2 \\
\hline Total & 58.616 & 6,0 & 55.714 & $-5,0$ & 51.577 & $-7,4$ & 61.128 & 18,5 & 62.084 & 1,6 \\
\hline
\end{tabular}

Fuente: SPEE.

En resumen, hasta el momento, la contratación de las personas con discapacidad presenta mejores resultados que la de los no discapacitados. El aumento observado en la contratación de las personas con discapacidad en los últimos años se debe al incremento de la contratación temporal, principalmente de los contratos eventuales y de interinidad, mientras que el uso de contratos indefinidos ha descendido, aunque no de forma tan intensa como en el caso de los traba- 
jadores sin discapacidad. El uso de contratos específicos para personas con discapacidad ha disminuido, quizás como consecuencia de los requisitos de mantenimiento del empleo más difíciles de cumplir en un entorno de crisis.

\subsubsection{La contratación en los Centros Especiales de Empleo}

Dentro de los datos de contratación de personas con discapacidad se engloban dos colectivos muy dispares, aquellos que prestan servicios en una empresa ordinaria y los que lo hacen en un CEE. Los escasos estudios sobre CEE coinciden en las diferencias entre el empleo protegido y el ordinario, lo que hace recomendable, en la medida de lo posible, separar en función del tipo de empresa el análisis de la contratación.

El objetivo de esta sección es cuantificar hasta que medida los resultados observados para la contratación de personas con discapacidad son consecuencia del empleo protegido. Además, en parte de este análisis se introduce una perspectiva autonómica pues la distribución de los CEE por el territorio español es diversa (véase Cueto et al. 2008).

Si atendemos al porcentaje que representan las contrataciones en CEE sobre el total de los contratos celebrados con personas con discapacidad (Cuadro 3.3), suponen en torno a la mitad. Además, esta cifra ha aumentado en el periodo considerando, pasando de un 47,5 por ciento en 2006 a un 66 por ciento en 2011. Por otra parte, se pueden apreciar diferencias sustanciales según la comunidad autónoma de referencia. En el País Vasco y Asturias, más de tres cuartas partes de las contrataciones a personas con discapacidad corresponden a CEE (en 2011, un 88,4 y un 80,8 por ciento, respectivamente), en otras como Melilla, Murcia, Baleares o Galicia la contratación en CEE no alcanza el 50 por ciento de la contratación total de personas con discapacidad.

Cuadro 3.3.

Contrataciones a personas con discapacidad por CCAA

\begin{tabular}{|l|c|c|c|c|c|c|c|c|c|c|c|c|}
\hline & \multicolumn{2}{|c|}{2006} & \multicolumn{2}{c|}{2007} & \multicolumn{2}{c|}{2008} & \multicolumn{2}{c|}{2009} & \multicolumn{2}{c|}{2010} & \multicolumn{2}{c|}{2011} \\
\cline { 2 - 13 } & Total & $\%$ CEE & Total & $\begin{array}{c}\% \\
\text { CEE }\end{array}$ & Total & $\begin{array}{c}\% \\
\text { CEE }\end{array}$ & Total & $\begin{array}{c}\% \\
\text { CEE }\end{array}$ & Total & $\begin{array}{c}\% \\
\text { CEE }\end{array}$ & Total & $\begin{array}{c}\% \\
\text { CEE }\end{array}$ \\
\hline Andalucía & 9.080 & 54,3 & 10.708 & 59,2 & 10.377 & 58,5 & 10.413 & 62,7 & 11.912 & 66,8 & 11.838 & 68,6 \\
\hline Aragón & 1.812 & 51,5 & 1.610 & 39,9 & 1.518 & 48,9 & 1.342 & 52,5 & 1.539 & 59,7 & 1.696 & 63,6 \\
\hline Asturias & 3.127 & 63,5 & 3.412 & 68,2 & 4.133 & 75,4 & 4.174 & 79,9 & 4.549 & 81,1 & 4.105 & 80,8 \\
\hline Baleares & 1.094 & 29,2 & 1.125 & 24,4 & 1.013 & 35,8 & 889 & 32,3 & 910 & 36,2 & 967 & 42,9 \\
\hline Canarias & 1.839 & 34,9 & 2.076 & 40,7 & 2.189 & 37,6 & 1.699 & 47,8 & 1.912 & 52,2 & 2.124 & 55,0 \\
\hline Cantabria & 851 & 56,4 & 960 & 54,0 & 834 & 57,1 & 764 & 67,5 & 954 & 73,4 & 895 & 72,5 \\
\hline Castilla La Mancha & 1.954 & 40,5 & 2.396 & 46,0 & 2.200 & 51,1 & 2.104 & 60,9 & 2.543 & 65,9 & 2.217 & 63,6 \\
\hline
\end{tabular}


Cuadro 3.3. (continuación)

Contrataciones a personas con discapacidad por CCAA

\begin{tabular}{|l|c|c|c|c|c|c|c|c|c|c|c|c|}
\hline & \multicolumn{2}{|c|}{2006} & \multicolumn{2}{c|}{2007} & \multicolumn{2}{c|}{2008} & \multicolumn{2}{c|}{2009} & \multicolumn{2}{c|}{2010} & \multicolumn{2}{c|}{2011} \\
\cline { 2 - 13 } & Total & \% CEE & Total & $\begin{array}{c}\% \\
\text { CEE }\end{array}$ & Total & $\begin{array}{c}\text { \% } \\
\text { CEE }\end{array}$ & Total & $\begin{array}{c}\% \\
\text { CEE }\end{array}$ & Total & $\begin{array}{c}\% \\
\text { CEE }\end{array}$ & Total & $\begin{array}{c}\% \\
\text { CEE }\end{array}$ \\
\hline Castilla y León & 3.243 & 57,8 & 3.329 & 56,8 & 3.387 & 62,5 & 3.132 & 67,2 & 3.640 & 72,3 & 3.655 & 72,6 \\
\hline Cataluña & 8.540 & 42,7 & 8.718 & 43,2 & 7.810 & 48,3 & 6.603 & 55,5 & 8.249 & 62,3 & 8.149 & 63,3 \\
\hline C. Valenciana & 5.413 & 36,6 & 5.696 & 36,1 & 4.784 & 39,2 & 4.531 & 45,8 & 5.445 & 53,0 & 5.136 & 55,4 \\
\hline Extremadura & 1.427 & 61,9 & 1.618 & 62,2 & 1.584 & 66,7 & 1.475 & 68,3 & 1.598 & 70,2 & 1.544 & 73,9 \\
\hline Galicia & 2.705 & 29,1 & 2.963 & 27,6 & 2.767 & 32,3 & 2.316 & 32,1 & 3.023 & 50,0 & 2.850 & 48,3 \\
\hline Madrid & 6.186 & 38,0 & 5.888 & 37,4 & 5.847 & 46,5 & 5.451 & 53,4 & 6.858 & 61,9 & 8.210 & 65,7 \\
\hline Murcia & 2.188 & 20,8 & 2.311 & 21,4 & 1.769 & 21,4 & 1.509 & 24,8 & 1.608 & 23,3 & 1.719 & 25,7 \\
\hline Navarra & 716 & 57,0 & 685 & 48,9 & 506 & 45,5 & 482 & 54,8 & 573 & 51,8 & 619 & 53,8 \\
\hline País Vasco & 4.543 & 76,8 & 4.460 & 78,4 & 4.337 & 81,5 & 4.158 & 83,7 & 5.199 & 86,4 & 5.757 & 88,4 \\
\hline La Rioja & 468 & 60,9 & 503 & 57,1 & 517 & 66,2 & 429 & 71,1 & 501 & 64,3 & 470 & 67,2 \\
\hline Ceuta & 61 & 41,0 & 71 & 52,1 & 90 & 67,8 & 59 & 72,9 & 54 & 50,0 & 55 & 52,7 \\
\hline Melilla & 70 & 21,4 & 84 & 14,3 & 49 & 12,2 & 44 & 11,4 & 59 & 16,9 & 54 & 20,4 \\
\hline Extranjero & 6 & 66,7 & 3 & 0,0 & 3 & 66,7 & 3 & 66,7 & 2 & 100,0 & 24 & 62,5 \\
\hline Total & 55.323 & 47,5 & 58.616 & 48,5 & 55.714 & 53,3 & 51.577 & 59,0 & 61.128 & 64,3 & 62.084 & 66,0 \\
\hline
\end{tabular}

Fuente: SPEE.

En cuanto a la evolución en el periodo señalado, mientras el número de contratos a personas con discapacidad ha aumentado un 12,2 por ciento, la cifra correspondiente a contratos en CEE lo ha hecho en un 55,8 por ciento. Por lo tanto si tenemos en cuenta únicamente los contratos celebrados con personas con discapacidad en empresas ordinarias estos se han reducido en un 27,3 por ciento en el periodo analizado.

En el Gráfico 3.4 podemos observar como a partir de 2007 desciende el número total de contratos registrados en el Servicio Público de Empleo. Sin embargo, si nos centramos en los contratos registrados con personas con discapacidad éstos han aumentado desde 2009, aunque entre 2010 y 2011 se mantienen relativamente constantes. Pero si desagregamos los contratos celebrados con personas con discapacidad entre los celebrados en empresas ordinarias o en CEE, observamos que la variación positiva de los contratos de personas con discapacidad se debe al aumento de los contratos en CEE, mientras que la contratación de personas con discapacidad en empresas ordinarias sigue una distribución muy similar a la del total de los contratos, incluso un poco inferior. 


\section{Gráfico 3.4.}

Evolución de los contratos según discapacidad

(Base 100, año 2006)

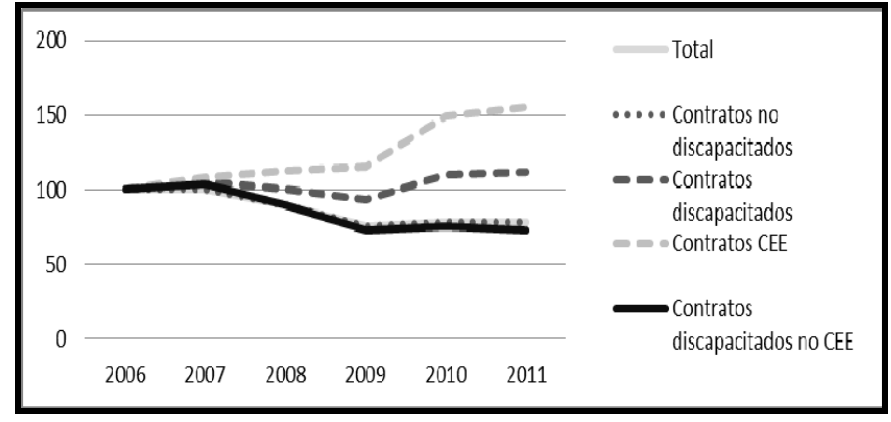

Fuente: SPEE.

Si analizamos únicamente los contratos indefinidos, Gráfico 3.5, observamos una tendencia similar pero con algunas variaciones. En este caso la evolución de los contratos indefinidos de las personas con discapacidad es similar a la de los contratos indefinidos en empresas ordinarias y aunque la tendencia desde 2007 ha sido negativa se ha estabilizado a partir de 2009, siendo a partir de entonces superior a la de contratos indefinidos. Por el contrario, los contratos indefinidos en CEE aumentaron hasta 2008 y posteriormente iniciaron un retroceso que se atenuó entre 2009 y 2010, que vuelve a coger impulso en 2011.

\section{Gráfico 3.5.}

Evolución de los contratos indefinidos

(Base 100, año 2006)

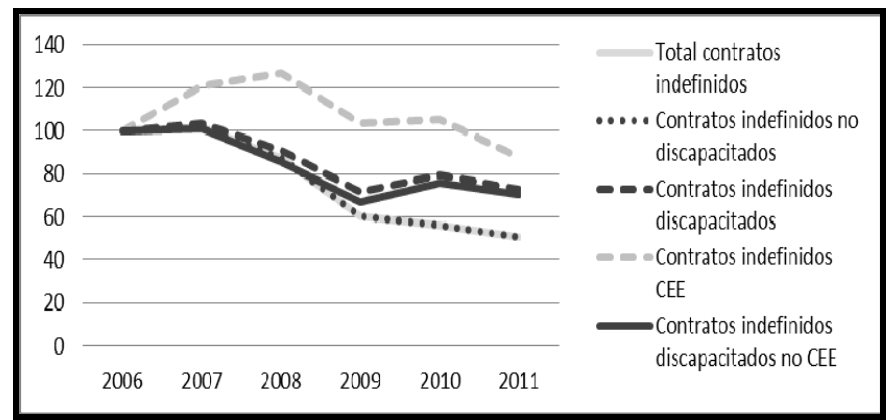

Fuente: SPEE.

Respecto a los contratos temporales registrados (Gráfico 3.6), los contratos a personas con discapacidad en empresas ordinarias siguen una distribución similar a la de los contratos de personas no discapacitadas, aunque el retroceso sufri- 
do desde 2007 ha tardado más en atenuarse que para los contratos temporales de los no discapacitados. Sin embargo, los contratos temporales en CEE han experimentado un fuerte incremento, principalmente entre 2009 y 2010.

Gráfico 3.6.

Evolución de los contratos temporales

(Base 100, año 2006)

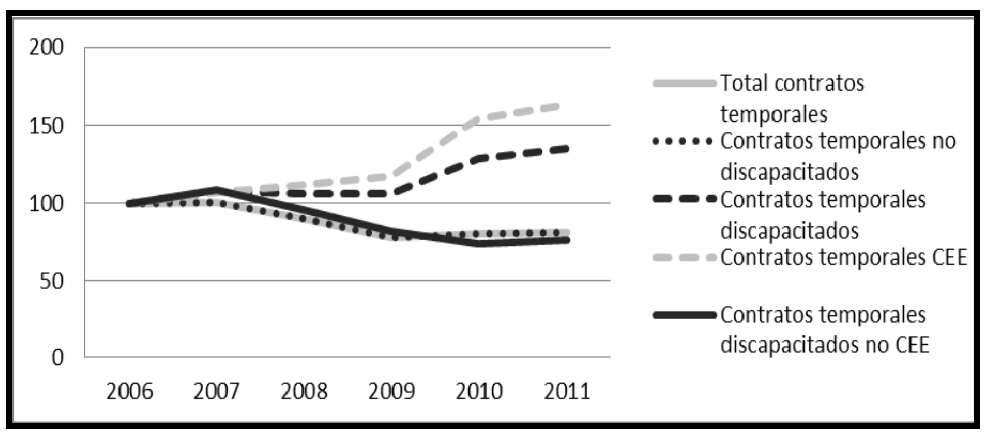

Fuente: SPEE.

Este incremento coincide con la ampliación transitoria de las subvenciones por mantenimiento del empleo en CEE, aprobada por la Ley 27/2009. Esta norma estableció entre el 10 de julio de 2009 y el 31 de diciembre 2010 (o el 31 de diciembre de 2011 en caso de discapacidad severa) que la subvención por mantenimiento del empleo en los CEE pasa del 50 por ciento al SMI al 75 por ciento, independientemente de la modalidad contractual.

A la hora de explicar el aumento en la contratación en CEE en un momento de crisis se debe tener en cuenta las diversas ayudas de las que son beneficiarios independientemente de que el contrato celebrado sea indefinido o temporal; ayudas para el mantenimiento de puestos de trabajo en CEE (bonificación, durante toda la vigencia del contrato, del $100 \%$ de la cuota empresarial a la Seguridad Social; subvención, durante toda la vigencia del contrato, del coste salarial correspondiente al puesto de trabajo ocupado por un discapacitado por un importe del 50\% del SMI; subvención para adaptación de puestos de trabajo en una cuantía no superior a 1.800 euros por persona contratada; subvención dirigida a equilibrar el presupuesto de aquellos CEE que carezcan de ánimo de lucro de hasta 1.500 euros por persona con discapacidad contratada) y ayudas a los proyectos generadores de empleo (para asistencia técnica; para sufragar la inversión fija en proyectos de interés social y para la subvención parcial de intereses de los préstamos). Además por cada nueva contratación con carácter indefinido o por la transformación en indefinidos de los contratos temporales el CEE recibe una subvención de entre 9.000 y 15.000 euros en función de la 
severidad de la discapacidad del trabajador y del porcentaje de trabajadores con discapacidad en la plantilla.

Si analizamos la contratación en CEE desagregada por meses, Cuadro 3.4, observamos que 2010 es el único año en el que en todos los meses se dio un incremento interanual de la contratación en $\mathrm{CEE}$, con un crecimiento anual de casi el 30 por ciento respecto a 2009. Durante ese año estuvo en vigor el incremento de la subvención por mantenimiento del empleo, cuyos efectos no se ven con tanta claridad en 2009 al ser una medida que se aprobó de forma retroactiva el 30 de diciembre de 2009 ni en 2011 donde solo permanecía vigente en caso de contratación a personas con discapacidad severa.

Cuadro 3.4.

Contratación en CEE por meses

\begin{tabular}{|c|c|c|c|c|c|c|c|c|c|c|c|}
\hline & 2006 & & 007 & & 008 & & 009 & & 010 & & 011 \\
\hline & Total & Total & $\begin{array}{c}\text { Variación } \\
\text { interanual } \\
(\%)\end{array}$ & Total & $\begin{array}{c}\text { Variación } \\
\text { interanual } \\
(\%)\end{array}$ & Total & $\begin{array}{c}\text { Variación } \\
\text { interanual } \\
(\%)\end{array}$ & Total & $\begin{array}{c}\text { Variación } \\
\text { interanual } \\
(\%)\end{array}$ & Total & $\begin{array}{c}\text { Variación } \\
\text { interanual } \\
(\%)\end{array}$ \\
\hline Enero & 2.069 & 2.194 & 6,0 & 2.545 & 16,0 & 2.236 & $-12,1$ & 2.645 & 18,3 & 2.990 & 13,0 \\
\hline Febrero & 1.904 & 2.196 & 15,3 & 2.353 & 7,1 & 1.918 & $-18,5$ & 2.623 & 36,8 & 3.122 & 19,0 \\
\hline Marzo & 2.274 & 2.440 & 7,3 & 2.227 & $-8,7$ & 2.056 & $-7,7$ & 3.093 & 50,4 & 3.485 & 12,7 \\
\hline Abril & 1.839 & 2.229 & 21,2 & 2.699 & 21,1 & 2.209 & $-18,2$ & 2.874 & 30,1 & 3.247 & 13,0 \\
\hline Mayo & 2.355 & 2.618 & 11,2 & 2.614 & $-0,2$ & 2.542 & $-2,8$ & 3.134 & 23,3 & 3.655 & 16,6 \\
\hline Junio & 2.445 & 2.603 & 6,5 & 2.581 & $-0,8$ & 2.859 & 10,8 & 3.660 & 28,0 & 4.137 & 13,0 \\
\hline Julio & 3.188 & 2.513 & $-21,2$ & 3.082 & 22,6 & 3.418 & 10,9 & 4.114 & 20,4 & 4.138 & 0,6 \\
\hline Agosto & 1.626 & 1.943 & 19,5 & 1.727 & $-11,1$ & 2.096 & 21,4 & 2.836 & 35,3 & 2.999 & 5,7 \\
\hline Septiembre & 2.175 & 2.218 & 2,0 & 2.860 & 28,9 & 2.746 & $-4,0$ & 3.818 & 39,0 & 3.607 & $-5,5$ \\
\hline Octubre & 2.396 & 2.968 & 23,9 & 3.007 & 1,3 & 3.003 & $-0,1$ & 3.695 & 23,0 & 3.217 & $-12,9$ \\
\hline Noviembre & 2.340 & 2.572 & 9,9 & 2.092 & $-18,7$ & 2.849 & 36,2 & 3.870 & 35,8 & 3.295 & $-14,9$ \\
\hline Diciembre & 1.679 & 1.944 & 15,8 & 1.919 & $-1,3$ & 2.510 & 30,8 & 2.975 & 18,5 & 3.078 & 3,5 \\
\hline Total & 26.290 & 28.438 & 8,2 & 29.706 & 4,5 & 30.442 & 2,5 & 39.337 & 29,2 & 40.970 & 4,2 \\
\hline
\end{tabular}

Fuente: SPEE. 
En el Cuadro 3.4 se muestra el tipo de contratación realizada a personas con discapacidad en 2011. Predomina con claridad el contrato temporal principalmente en los CEE (76,1 por ciento del total para el total de PCD y 94,4 por ciento para los CEE). En cuanto a la contratación total de personas con discapacidad, el porcentaje de contratos indefinidos para minusválidos supone aproximadamente la mitad del total de contratos indefinidos, también con una importante diferencia por comunidades autónomas; Murcia y La Rioja presentan los valores más elevados de contratos indefinidos para minusválidos (aproximadamente el 65 por ciento) y Andalucía y País Vasco presentan los valores más bajos (sobre el 33 por ciento); mientras que este tipo de contrato no tiene presencia en los CEE.

Si atendemos a la forma de contratación de los CEE (Cuadro 3.5), observamos que predomina la contratación temporal, al igual que ocurre con las contrataciones de personas con discapacidad en general, aunque utilizando fundamentalmente figuras ordinarias (es decir, no se trata de contratos específicos para personas con discapacidad, aunque pueden estar incentivados). Se debe tener en cuenta que en los CEE cualquier tipo de contrato celebrado con una persona con discapacidad está subvencionado; por lo tanto no necesitan celebrar un contrato específico para personas con discapacidad para recibir incentivos a la contratación, por lo que es esperable el menor uso del contrato temporal para minusválidos, de duración mínima de 1 año, que puede resultar menos atractivo que otros contratos temporales. ${ }^{6}$ De ahí que no se observe que los CEE utilicen las figuras contractuales específicas para personas con discapacidad.

Dado que uno de los objetivos de los CEE es fomentar el tránsito al mercado de trabajo ordinario, una mayor contratación temporal no tiene porqué reflejar mayor precariedad laboral, sino únicamente la idea de que, en un momento dado, los trabajadores abandonarán el CEE para trabajar en una empresa ordinaria. Sin embargo, si, tal y como indica la literatura, no parece que los CEE cumplan esta función, el volumen de contratación temporal utilizada podría estar indicando una elevada rotación ${ }^{7}$.

\footnotetext{
${ }^{6}$ Para más información sobre modalidades contractuales y bonificaciones a la contratación consultar Malo et al. (2009a) y Cueto et al. (2008).

${ }^{7}$ Se debe tener en cuenta que tanto en el convenio colectivo estatal de CEE, como en la mayoría de convenios colectivos de empresa aplicables a estos centros se regula la posibilidad de solicitar una excedencia para la incorporación a la empresa ordinaria, con reserva del puesto de trabajo; sin embargo, algunos establecen la obligación de que el trabajador tenga una antigüedad de al menos un año en el CEE, lo que puede verse dificultado por la alta rotación.
} 
Cuadro 3.5 .

Contrataciones a personas con discapacidad según tipo de contrato (2011)

\begin{tabular}{|c|c|c|c|c|c|c|c|c|}
\hline & \multicolumn{4}{|c|}{ Total personas con discapacidad } & \multicolumn{4}{|c|}{ CEE } \\
\hline & \multicolumn{2}{|c|}{ Indefinidos } & \multicolumn{2}{|c|}{ Temporales } & \multicolumn{2}{|c|}{ Indefinidos } & \multicolumn{2}{|c|}{ Temporales } \\
\hline & Total & $\begin{array}{c}\text { \% minusvál. } \\
\text { /total }\end{array}$ & Total & \begin{tabular}{|c|}
$\begin{array}{c}\% \text { minusvál. } \\
\text { /total }\end{array}$ \\
\end{tabular} & Total & $\begin{array}{c}\% \text { minusvál. } \\
\text { /total }^{*}\end{array}$ & Total & $\begin{array}{c}\% \text { minusvál. } \\
\text { /total }\end{array}$ \\
\hline Andalucía & 2.598 & 33,0 & 9.240 & 30,3 & 790 & 0,0 & 7.333 & 11,6 \\
\hline Aragón & 439 & 53,3 & 1.257 & 32,0 & 73 & 0,0 & 1.006 & 15,3 \\
\hline Asturias & 568 & 54,0 & 3.537 & 11,3 & 109 & 0,0 & 3.206 & 3,7 \\
\hline Baleares & 343 & 65,9 & 624 & 45,0 & 46 & 0,0 & 369 & 13,6 \\
\hline Canarias & 494 & 53,0 & 1.630 & 43,0 & 46 & 0,0 & 1.122 & 20,3 \\
\hline Cantabria & 181 & 58,0 & 714 & 18,3 & 5 & 0,0 & 644 & 8,1 \\
\hline C. La Mancha & 599 & 49,9 & 1.618 & 32,8 & 89 & 0,0 & 1.322 & 14,0 \\
\hline Castilla y León & 846 & 43,5 & 2.809 & 27,1 & 150 & 0,0 & 2.505 & 11,5 \\
\hline Cataluña & 2.377 & 47,8 & 5.772 & 46,7 & 256 & 0,0 & 4.905 & 28,0 \\
\hline C. Valenciana & 1.410 & 57,3 & 3.726 & 39,2 & 91 & 0,0 & 2.756 & 15,6 \\
\hline Extremadura & 302 & 53,6 & 1.242 & 23,8 & 38 & 0,0 & 1.103 & 11,2 \\
\hline Galicia & 796 & 56,7 & 2.054 & 44,4 & 60 & 0,0 & 1.317 & 9,9 \\
\hline Madrid & 2.213 & 51,4 & 5.997 & 34,0 & 436 & 0,0 & 4.957 & 15,2 \\
\hline Murcia & 537 & 65,0 & 1.182 & 61,3 & 24 & 0,0 & 417 & 17,5 \\
\hline Navarra & 211 & 51,7 & 408 & 47,5 & 2 & 0,0 & 331 & 23,9 \\
\hline País Vasco & 747 & 33,3 & 5.010 & 18,7 & 78 & 0,0 & 5.013 & 12,8 \\
\hline La Rioja & 101 & 64,4 & 369 & 33,1 & 8 & 0,0 & 308 & 17,5 \\
\hline Ceuta & 18 & 88,9 & 37 & 27,0 & 0 & 0,0 & 27 & 0,0 \\
\hline Melilla & 30 & 83,3 & 24 & 62,5 & 0 & 0,0 & 9 & 22,2 \\
\hline Extranjero & 17 & 0,0 & 7 & 0,0 & 0 & 0,0 & 14 & 0,0 \\
\hline Total & 14.827 & 48,3 & 47.257 & 32,6 & 2.306 & 0,0 & 38.664 & 14,5 \\
\hline
\end{tabular}

* El valor de esta columna es 0 para todas las CCAA porque ningún CEE ha celebrado contratos indefinidos específicos para personas con discapacidad. Esto no implica que no los puedan realizar, sino que no los utilizan. Se debe tener en cuenta que todos los contratos celebrados por un CEE están bonificados independientemente de su modalidad, por lo que esta categoría específica no implica ventajas añadidas.

Fuente: SPEE.

El aumento de la contratación en los CEE está relacionado con el fuerte aumento del número de CEE en el periodo estudiado a pesar de la crisis (que ha pasado de 1.573 en 2006 a 1.775 en 2008 y 1.900 en $2009^{8}$ ). Este aumento nos puede llevar a pensar que los CEE no se han visto afectados por la crisis. Esta

\footnotetext{
${ }^{8}$ Según Malo et al. (2009) y FEACEM (http://www.feacem.es/FEACEM/CEE/cifras.htm).
} 
situación puede ser debida, además de al amplio número de subvenciones y ayudas de las que disponen, al aumento de la utilización por parte de las empresas ordinarias de las medidas alternativas al cumplimiento de la cuota de reserva; principalmente en cuanto al uso de los servicios de un $\mathrm{CEE}^{9}$ para el suministro de materias o bienes o para la prestación de servicios ajenos y accesorios a la actividad normal de la empresa. Teniendo en cuenta que los CEE concentran su actividad en los servicios, transportes y comunicaciones (Malo et al., 2009), que las empresas de más de 50 trabajadores deben cumplir la cuota de reserva de personas con discapacidad o las medidas alternativas y que en los últimos años ha aumentado la utilización de estas medidas alternativas podríamos pensar que el auge de los CEE está relacionado con la preferencia de las empresas ordinaria por celebrar contratos mercantiles con estos centros y externalizar actividades como la limpieza, el mantenimiento o la atención al cliente. Esta situación parece ser opuesta al espíritu de la LISMI (que busca primar el empleo ordinario sobre el protegido) dándose la situación en el que el espíritu de una ley busca un objetivo mientras que su aplicación (y la de sus normas de desarrollo) van en sentido contrario.

En resumen, tras el aumento de la contratación de las personas con discapacidad se encuentra el aumento de la contratación en CEE, más concretamente, el aumento de la contratación temporal en CEE. Además, la incidencia de la contratación en CEE sobre el total de contrataciones a personas con discapacidad presenta diferencias regionales (al igual que la presenta la implantación territorial de este tipo de centros). El hecho de que la contratación en estos centros haya aumentado en un momento de fuerte crisis económica parece indicar la efectividad de las políticas destinadas a fomentar el empleo de las personas con discapacidad en CEE (pero no los incentivos a la contratación indefinida, sino las ayudas al mantenimiento del empleo que son independientes del tipo de contrato y cuyo aumento coincide temporalmente con el aumento de la contratación en CEE). Sin embargo, sería conveniente que las Administraciones Públicas analicen si este incremento es en detrimento de la contratación de personas con discapacidad en el mercado de trabajo ordinario y si es conveniente modificar las políticas destinadas a este colectivo.

\section{CONCLUSIONES}

Dentro de un escenario de crisis como el actual el empleo de las personas con discapacidad ha sufrido importantes cambios que se esconden tras el moderado (al menos respecto a los no discapacitados) ascenso de su tasa de desempleo.

\footnotetext{
${ }^{9}$ Según Malo et al. (2009) las empresas ordinarias que utilizan esta medida alternativa se han duplicado entre 2001 y 2006 (del 5,95 al 12,25 por ciento).
} 
La contratación de las personas con discapacidad en España ha aumentado durante lo que llevamos de recesión; sin embargo, tras ese aumento se encuentra un aumento de las contrataciones en CEE en detrimento de las contrataciones en empresas ordinarias. Además, en general, el aumento de la contratación de las personas con discapacidad se debe al aumento de la contratación temporal. En este periodo ha disminuido el uso de figuras contractuales específicas para personas con discapacidad (contratos bonificados) tanto temporales como indefinidas.

Los contratos celebrados por CEE casi se han duplicado desde 2006 y el incremento en la contratación se ha dado en los contratos temporales. Al igual que en el total de los contratos a personas con discapacidad observamos una escasa utilización de figuras específicas debido a que todas las contrataciones en CEE están bonificadas independientemente de su modalidad y duración.

Cómo se ha indicado anteriormente esta elevada temporalidad podría estar relacionada con la función de estos centros de ser un puente hacia el mercado de trabajo ordinario, sin embargo los escasos análisis al respecto nos hacen pensar que este objetivo no se cumple por lo que probablemente estemos ante una mayor precarización laboral de este colectivo. Los datos existentes nos llevan a pensar que lo que se está produciendo es un trasvase de personas con discapacidad en la empresa ordinaria hacia los CEE debido al aumento de la sustitución del cumplimiento de la cuota de reserva por las medidas alternativas.

No obstante, y esto es una novedad en la literatura sobre el tema, los datos analizados también nos permiten concluir que los CEE pueden desempeñar un papel de "refugio de empleo" para las personas con discapacidad, evitando así que pierdan el contacto con el mercado de trabajo durante las crisis (incluso en una recesión tan dura como ésta). Como continuación natural de este trabajo será necesario analizar que ocurre con el empleo en estos centros una vez que la crisis empiece a remitir; para comprobar si esta tendencia se mantiene o se modifica.

Sin embargo, en general, la literatura existente parece compartir la idea de que el empleo protegido no es un empleo con las mismas características que el ordinario, sino un empleo de menor calidad; por lo que es posible que las políticas implementadas alcancen un objetivo distinto al original.

Esto, unido a los resultados obtenidos por Malo y Muñóz-Bullón (2006), hacen recomendable un rediseño de las políticas activas destinadas a las personas con discapacidad; ya sea mediante una reformulación de objetivos, más acordes con los resultados realmente alcanzados, o mediante una transformación más profunda de las medidas aplicadas a este colectivo. 


\section{REFERENCIAS BIBLIOGRÁFICAS}

BALDWIN, M. y JOHNSON, W.G. (1992): "Estimating the Employment Effects of Wage Discrimination", The Review of Economics and Statistics, 74, pp. 446-455.

BALDWIN, M. Y JOHNSON, W.G. (1995): "Labor market discrimination against women with disabilities", Industrial Relations, 34 (4), pp. 555-577.

BALDWIN, M. Y JOHNSON, W.G. (2000), "Labor market discrimination against men with disabilities in the year of the ADA", Southern Economic Journal, 66 (3), p. 548-566.

BALDWIN, M.L. y SCHUMACHER, E.J. (2002): “A note on job mobility among workers with disabilities" Industrial Relations, 41(3), pp. 430-441.

BROWN, C.L. Y EMERY, J.C.H. (2008): "The Impact of Disability on Earnings and Labour Force Participation in Canada: Evidence from the 2001 PALS". Department of Economics, University of Calgary. Working Paper 2008-26. Disponible en http://econ.ucalgary.ca/sites/econ.ucalgary.ca/files/publications/ PALSworkingpaper2008.pdf [Último acceso: Marzo 2012]

CCOO (2009): "Los Centros especiales de empleo incrementan del $50 \%$ al $75 \%$ del salario mínimo las ayudas por contrato de trabajo, pero mantienen congelados los salarios". CCOO informa de 9 de diciembre de 2009.

CCOO (2010): "CCOO denuncia que las patronales del sector de la discapacidad proponen una rebaja salarial de los trabajadores". CCOO informa de 13 de marzo de 2010.

CUETO, B., MALO, M.A., RODRÍGUEZ, V. y FRANCOS, C. (2008): Trayectorias laborales de las personas con discapacidad y Centros especiales de empleo: análisis empírico con la Muestra de Vidas Laborales. Disponible en: http://www.seg-social.es/prdi00/groups/public/documents/binario/119779.pdf [Último acceso: Enero 2012]

CUETO, B., MALO, M.A. y RODRÍGUEZ, V. (2012): “La brecha de participación laboral de las personas con discapacidad en España. Un análisis con la EDAD-2008". Cuadernos del Mercado de Trabajo, Núm. 8, pp. 20-27. Próxima publicación

DÁVILA, C.D. (2003): Perfil educativo y participación laboral de los jóvenes con discapacidad. Comunicación en las XII Jornadas de la Asociación de Economía de la Educación. Getafe, 18 y 19 septiembre 2003.

DÁVILA, C.D. (2006): "Discapacidad y género. Un estudio de participación en el mercado de trabajo español”. Moneda y Crédito, Núm. 223, pp.127-158.

DÁVILA, C.D. y MALO, M.A. (2006): "Género, discapacidad y posición familiar: la participación laboral de las mujeres con discapacidad". Cuadernos Aragoneses de Economía, 16(1), pp. 61-82.

DELSEN, L. (2001): "Economic study of special employment of disabled people in the European Union”. Paper EG0123. University of Nijmegen. 
ESTEBAN, R., PÉREZ, F. (Directores) (2009): "La aplicación del Derecho del Trabajo en los Centros especiales de empleo". Valencia: Editorial Tirant Lo Blanch. Monografías 643.

GANNON, B. y NOLAN, B. (2004): "Disability and labour market participation", Equality Studies Unit Report.

GANNON, B. y NOLAN, B. (2007): "Transitions in Disability and Work". Estudios de Economía Aplicada. 25(2), pp.447-472.

HUMER, B.; WUELLRICH, J. P. Y ZWEIMÜLLER, J. (2007): "Integrating severely disabled individuals into the Labour Market: the Austrian case“. IZA Discussion Paper, Núm. 2649. Disponible en http://ftp.iza.org/dp2649.pdf [Último acceso: Marzo 2012]

IVIE (2010): "Capital humano y empleo en tiempo de crisis". Capital Humano, № 118. Bancaja. Disponible en: http://www.ivie.es/downloads/docs/ch/ch122.pdf [Último acceso: Enero 2012]

JOHNSON, W. y LAMBRINOS, J. (1985), "Wage discrimination against handicapped men and women", Journal of Human Resources, 20(2), pp. 264-277.

JONES, M., LATREILLE, P. Y SLOANE, P. (2006): "Disability, gender, and the British labour market", Oxford Economic Papers, 58 (3); pp. 407-449.

JONES, M.K.; LATREILLE, P.L. y SLOANE, P.J. (2007): "Disability and work: a review of the British evidence", Estudios de Economía Aplicada, 25(2), pp. 473-498.

LÓPEZ, C.M. y SECO, E. (2005): "Discapacidad y empleo en España: su visibilidad" INNOVAR Revista de Ciencias Administrativas y Sociales, Julio a Diciembre de 2005; pp. 59-72.

MALO, M.A. (2001): "Discapacidad y participación en el mercado de trabajo", capítulo de Condiciones de vida en España y en Europa, Instituto Nacional de Estadística, Madrid, pp. 281-306.

MALO, M.A. (2003): "Las personas con discapacidad en el mercado de trabajo español". Revista del Ministerio de Trabajo y Asuntos Sociales, Núm. 46, pp. 99-126.

MALO, M.A. (2004): “¿Cómo afectan las discapacidades a la probabilidad de ser activo en España? Un análisis empírico con datos de la Encuesta sobre Discapacidades, Deficiencias y Estado de la Salud de 1999". Cuadernos de Economía, Vol. 27, pp. 75-108.

MALO, M.A. y MUÑOZ-BULLÓN, F. (2006): "Employment promotion measures and the quality of the job match for persons with disabilities". Hacienda Pública Española / Revista de Economía Pública, 179, pp.79-111.

MALO, M.A. (2007): "La definición de la discapacidad en la investigación económica: Una reflexión necesaria sobre qué características debería cumplir". Estudios de Economía Aplicada, 25(2), pp.407-428.

MALO, M.A., CUETO, B., DÁVILA, D., PAGÁN, R. y RODRÍGUEZ, V. (2009): "Informe de Evaluación sobre la política de bonificación de cuotas para las personas con discapacidad en situación de desempleo". Agencia de 
Evaluación y Calidad. Disponible en: http://www.aeval.es/comun/pdf/ evaluaciones/E19-2009.pdf [Último acceso: Enero 2012]

MALO, M.A. Y PAGÁN, R. (2012), "Wage differentials and disability: Discrimination and/or lower productivity?", International Labour Review, (forthcoming).

MEDINA, E. HERRARTE, A. Y VICÉNS, J. (2010): "Inmigración y desempleo en España: impacto de la crisis económica", Información Comercial Española. Revista de Economía, Núm. 854, pp. 37-48

OCDE (1992): "Employment policies for people with disabilities - Report by an evaluation panel" OECD Labour Market and Social Policy Occasional Papers Núm. 8.

PAGÁN, R. (2007): "Dynamic analysis of disability in Spain using the European Community Household Panel". Estudios de Economía Aplicada, 25(2), pp. 499-522.

PAGÁN, R. (2011a): "Ageing and disability: Job satisfaction differentials across Europe”, Social Science \& Medicine, 72 (2), pp. 206-215.

PAGÁN, R. (2011b): "Self-employment and job satisfaction: Evidence for older people with disabilities in Europe", European Journal of Ageing, 8 (3), pp. 177-187.

PAGÁN, R. (2012): "Transitions to part-time work at older ages: The case of people with disabilities in Europe", Disability and Society, 27 (1), pp. 95-115.

PAGÁN, R. Y MALO, M.A (2009), "Job satisfaction and disability: Lower expectations about jobs or a matter of health". Spanish Economic Review, 11(1), pp.51-74.

PASCUAL, M. y CANTARERO, D. (2007): "Socio-demographic determinants of disabled people: An empirical approach based on the European Community Household Panel". Journal of Socio-Economics, 36(2), pp. 275-287

RUBIO, F.J. (2003): "La construcción social del empleo protegido: los Centros especiales de empleo" Nómadas Núm.8 - Universidad Complutense de Madrid

VILLA, N. (2003): "Situación laboral de las personas con discapacidad en España". Revista Complutense de Educación. 14(2), pp.393-424.

VISIER, L. (1998): "Relaciones laborales en los sistemas de trabajo protegido para personas minusválidas" Revista internacional del Trabajo, 117(3); pp. 371-390.

ZWINKELS, W. (2001). "The employment situation of people whit disabilities in the European Union." Research paper, EIM Business and Policy Research. 
